\title{
Idiopathic Accessory Hemiazygos Vein Aneurysm with an Incidental Mediastinal Mass
}

\author{
Yoichi Imori ${ }^{1}$, Hajime Fujimoto ${ }^{2}$, Shigeo Hanada ${ }^{1}$, Hironori Uruga ${ }^{1}$, Hisashi Takaya ${ }^{1}$, \\ Atushi Miyamoto ${ }^{1}$, Nasa Morokawa ${ }^{1}$, Noboru Shindo ${ }^{3}$ and Kazuma Kishi ${ }^{1,4}$
}

\begin{abstract}
We herein report the case of an idiopathic aneurysm of the accessory hemiazygos vein diagnosed by contrast-enhanced computed tomography (CT) and angiography. The patient was asymptomatic, and a posterior mediastinal mass was incidentally found on CT. Because endoscopic ultrasonography showed no thrombus formation in the aneurysm, the patient was therefore managed conservatively.
\end{abstract}

Key words: azygos vein system, aneurysm, accessory hemiazygos vein, mediastinal mass, incidental mediastinal mass, computed tomography

(Intern Med 51: 2347-2349, 2012)

(DOI: 10.2169/internalmedicine.51.8011)

\section{Introduction}

Idiopathic aneurysms of the azygos vein system (azygos vein, hemiazygos vein, and accessory hemiazygos vein) are rare, and there is no standard treatment (1-5). We herein report a case of an asymptomatic aneurysm of the accessory hemiazygos vein that was diagnosed based on the findings of contrast-enhanced computed tomography (CT) and angiography.

\section{Case Report}

A 62-year-old woman was admitted to our hospital because of a mediastinal mass detected on a CT screen for lung cancer. The patient was asymptomatic, and there was no remarkable past medical history except for a duodenal ulcer. No significant trauma had been recorded. A physical examination and blood analysis including a hemogram, differential cell count, and the patient's hepatic and renal function were normal. A chest radiograph was normal, and no signs of a posterior mediastinal mass were noted (Fig. 1). However, a non-enhanced CT scan showed a triangularshaped posterior mediastinal mass $20 \times 15 \mathrm{~mm}$ in size between the third thoracic vertebra and the descending aorta
(Fig. 2A). Contrast-enhanced CT revealed the presence of a homogeneously enhanced mass close to the accessory hemiazygos vein (Fig. 2B). Endoscopic ultrasonography (EUS) showed an anechoic mass adjacent to the accessory hemiazygos vein with a blood flow, thus suggesting the mass to not be a thrombotic aneurysm (Fig. 3). We performed angiography of the azygos vein system, and a diagnosis of idiopathic accessory hemiazygos vein aneurysm was made (Fig. 4). Because the patient was asymptomatic and the endoscopic ultrasonography suggested a non-thrombosed aneurysm, we therefore followed the patient conservatively.

\section{Discussion}

We herein described a case of idiopathic aneurysm of the accessory hemiazygos vein that was incidentally found on CT. An idiopathic aneurysm of the azygos vein system is a rare condition, and an accessory hemiazygos vein aneurysm is exceedingly rare, with only one case reported previously (6). Most cases of azygos vein aneurysms have occurred as a result of congestive heart failure, obstruction of the inferior vena cava by tumors or other causes, or malformation of the inferior vena cava, including partial or total agenesis (1). In our case, none of these were determined to be the cause, and the accessory hemiazygos vein aneurysm

${ }^{1}$ Department of Respiratory Medicine, Respiratory Center, Japan, ${ }^{2}$ Department of Cardiovascular Medicine, Toranomon Hospital, Japan, ${ }^{3}$ Department of Diagnostic Radiology, Toranomon Hospital, Japan and ${ }^{4}$ Okinaka Memorial Institute for Medical Research, Japan

Received for publication April 19, 2012; Accepted for publication May 29, 2012

Correspondence to Dr. Yoichi Imori, yoichiimori@me.com 


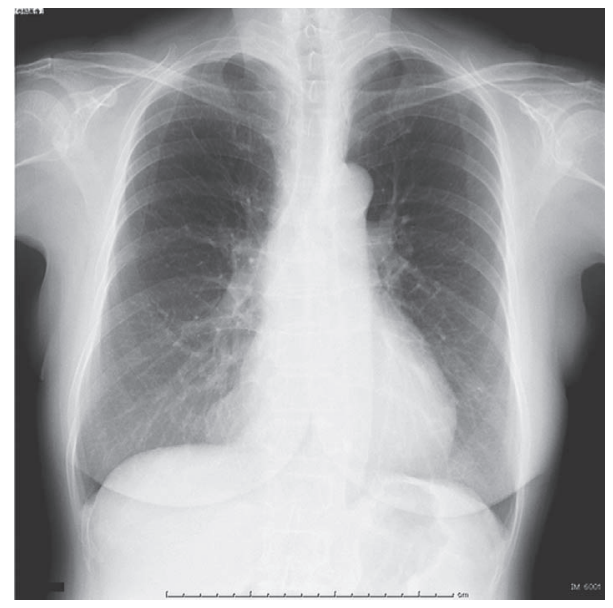

Figure 1. The chest radiograph was normal.
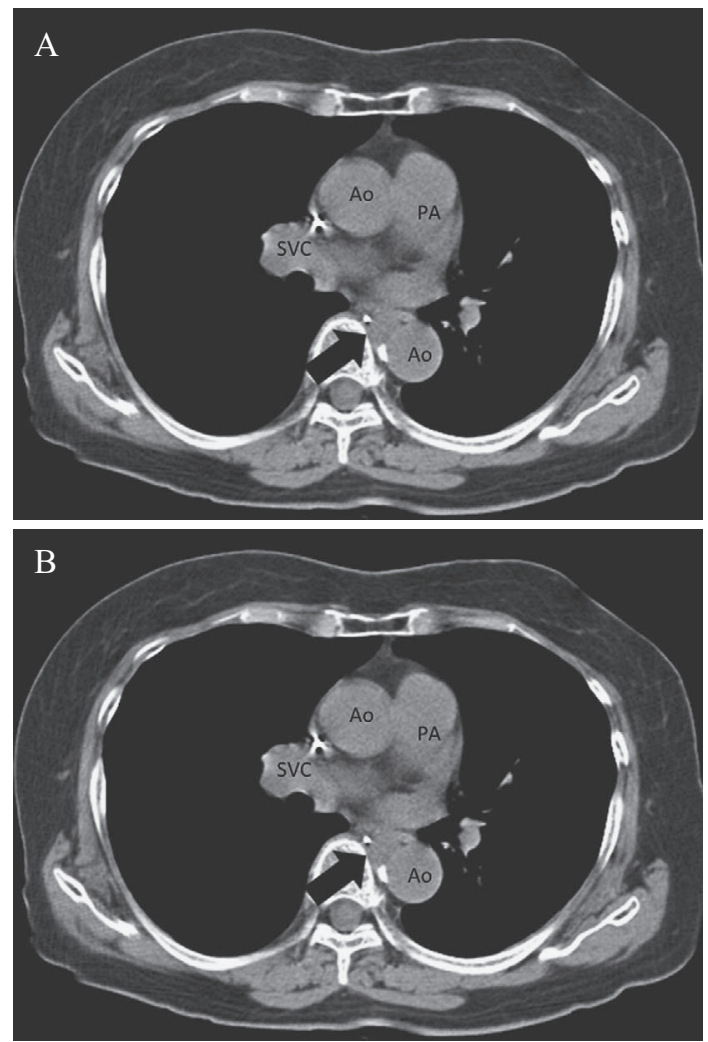

Figures 2. A: The non-enhanced computed tomographic scan showed a posterior mediastinal mass between the third thoracic vertebra and descending aorta (arrow), and B: a contrast-enhanced computed tomographic scan showed a homogeneously enhanced mass close to the accessory hemiazygos vein (arrow). SVC: superior vena cava, AZ: azygos vein, Ao: aorta, PA: pulmonary artery

was considered to be idiopathic.

An azygos vein aneurysm is often asymptomatic and tends to be detected incidentally on chest radiography. The abnormality appearing on a chest radiograph is often nonspecific, and may appear as a prominent azygos vein or mediastinal mass. However, there were no particular findings in the chest radiograph in our case, and CT screening for lung

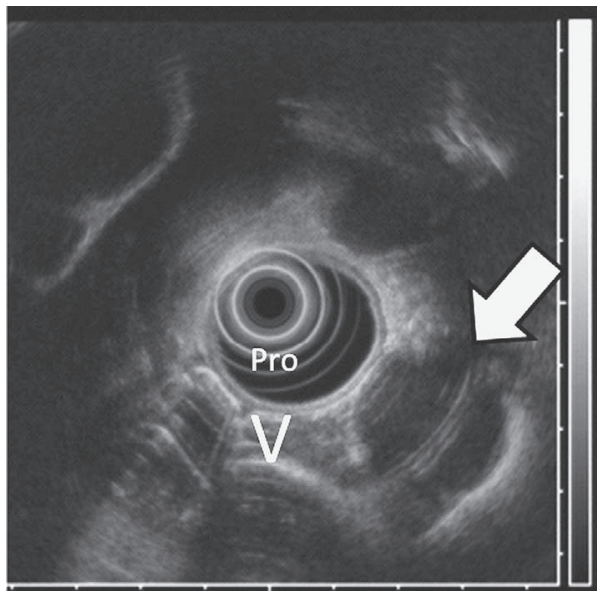

Figure 3. The $360^{\circ}$ radial scanning endoscopic ultrasonogram showed an anechoic mass adjacent to the accessory hemiazygos vein with blood flow (arrow). Pro: echo probe, V: vertebra

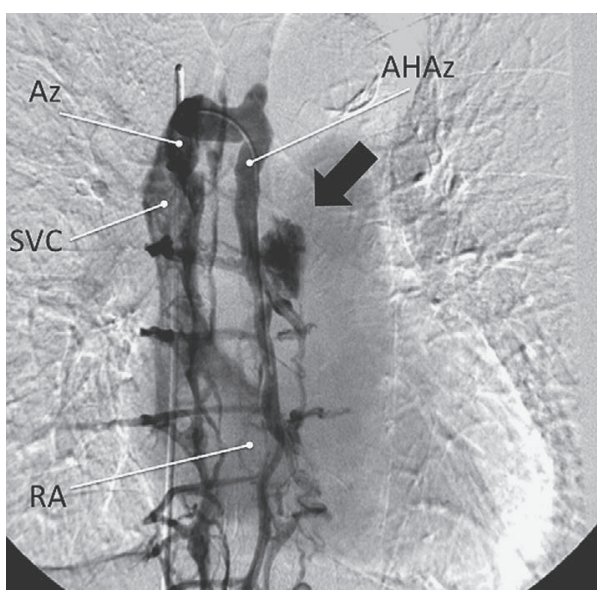

Figure 4. The angiogram of the azygos vein revealed the accessory azygos vein with an aneurysm (arrow). SVC: superior vena cava, AZ: azygos vein, AHAz: accessory hemiazygos vein, RA: right atrium

cancer detected a posterior mediastinal mass.

Contrast-enhanced CT provides useful data for the diagnosis, because it helps to confirm the vascular nature of the lesion and distinguishes it from other mediastinal tumors $(2,3)$. In our case, angiography, followed by contrastenhanced CT confirmed the diagnosis of an accessory hemiazygos vein aneurysm.

In previous reports, transesophageal echocardiography (TEE) has been reported to be useful for the diagnosis of azygos vein aneurysms (1). We also performed TEE in the present case, but could not clearly identify the accessory hemiazygos vein aneurysm because of the probe position, angle, and manipulation problems. However, endoscopic ultrasonography clearly revealed the aneurysm with a blood flow. We therefore assumed that the vertebra had obstructed the echo beam from TEE convex probe because the hemiazygos vein aneurysm was close to the vertebra, whereas $360^{\circ}$ radial scanning EUS was able to show the lesion. 
A standard therapeutic strategy for aneurysm of the azygos vein system has not been established. The aneurysm may result in rupture, hemorrhage, or pulmonary thromboembolism, but the probability of such critical outcomes is unclear (7-9). Chu et al. stated that conservative management may be reasonable if the patients are asymptomatic (7). They also stated that surgical treatment might be indicated if patients complain of symptoms related to the aneurysm. Accordingly, we decided to follow the patient symptoms and checked the aneurysm size and presence of intraluminal thrombus of the aneurysm by contrast-enhanced CT or EUS depending on the situation.

To the best of our knowledge, this is the second report of an idiopathic accessory hemiazygos vein aneurysm, which is considered to be one of the differential diagnoses of a posterior mediastinal mass.

The authors state that they have no Conflict of Interest (COI).

\section{References}

1. Lena H, Desrues B, Heresbach D, et al. Azygos vein aneurysm: contribution of transesophageal echography. Ann Thorac Surg 61: 1253-1255, 1996.

2. Gomez MA, Delhommais A, Presicci PF, Besson M, Roger R, Alison D. Partial thrombosis of an idiopathic azygos vein aneurysm. Br J Radiol 77: 342-343, 2004.

3. Gallego M, Mirapeix RM, Castaner E, Domingo C, Mata JM, Marin A. Idiopathic azygos vein aneurysm: a rare cause of mediastinal mass. Thorax 54: 653-655, 1999.

4. Seebauer L, Prauer HW, Gmeinwieser J, Sebening F. A mediastinal tumor simulated by a sacculated aneurysm of the azygos vein. Thorac Cardiovasc Surg 37: 112-114, 1989.

5. Rockoff SD, Druy EM. Tortuous azygos arch simulating a pulmonary lesion. AJR Am J Roentgenol 138: 577-579, 1982.

6. Vishnevskii AA, Mazhorov VA, Pozdeev IV, Nikoladze GD. [Thrombosed aneurysm of the accessory hemiazygos vein simulating neurinoma of the posterior mediastinum]. Vestn Khir Im I I Grek 142: 49-50, 1989.

7. Chiu SS, Lau S, Kam CK. Azygous vein aneurysm: CT scan follow-up. J Thorac Imaging 21: 66-68, 2006.

8. Watanabe A, Kusajima K, Aisaka N, Sugawara H, Tsunematsu K. Idiopathic saccular azygos vein aneurysm. Ann Thorac Surg 65: 1459-1461, 1998.

9. Ishikura H, Kimura S, Fukumura Y, Ohtani T. Resection of an azygos vein aneurysm with thrombosis. Gen Thorac Cardiovasc Surg 58: 209-211, 2010.

(C) 2012 The Japanese Society of Internal Medicine http://www.naika.or.jp/imonline/index.html 